\title{
An Evidence-Based Herbal Supplement for The Control of Metabolic Syndrome
}

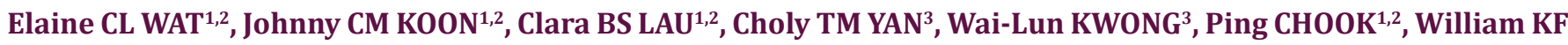 \\ CHENG $^{1,2}$ and Ping-Chung LEUNG ${ }^{1,2 *}$ \\ ${ }^{1}$ Institute of Chinese Medicine, The Chinese University of Hong Kong, Hong Kong \\ ${ }^{2}$ State Key Laboratory of Research on Bioactivities and Clinical Applications of Medicinal Plants (The Chinese University of Hong Kong) \\ ${ }^{3}$ Royal Medic Research and Development Center, Hong Kong Science Park, Hong Kong
}

*Corresponding author: Ping-Chung LEUNG, 5/F School of Public Health Building, Prince of Wales Hospital, Shatin, Hong Kong

\begin{abstract}
Background: Metabolic Syndrome (MS): Overweight, Obesity, Hypertension, Hyperglycemia and Hypercholesterolemia, are generally accepted today as clinical signals leading to cardiovascular diseases. Control of MS is therefore of a common health concern. While drug treatment is yet not available or may not be creditable, developing an effective health supplement against MS is highly justified.

Methods: A herbal formula composed of four herbs known to have anti MS pathological effects was used for in vivo and in vitro biological researches to verify its pharmacological effect, and subsequent pilot clinical trial.

Results: In vitro study: Adipocyte viability and cholesterol uptake, liver cell viability and anti-glycaemia effects, all gave positive results of good control. In vivo study testing herbal formula's effects on obese mice also showed very promising results. In clinical trial, measurements of body weight, body circumferences, BMI, as well as liver fibrosis, all showed good responses after the herbal formula consumption.

Conclusion: Our efforts on the creation of an Evidence-Based Specific Supplement for the control of Metabolic Syndrome have harvested highly positive data in the laboratory. The subsequent 3 months' pilot clinical trial showed positive data on the control of blood lipids, general body measurements and liver steatosis.
\end{abstract}

Keywords: Herbal Supplement; Metabolic Syndrome (MS); Herbal Formula; Pre-Clinical Study; Pilot Clinical Trial

\section{Introduction}

As cardiovascular diseases have become dominant causes of mortality, other related pathological presentations are gaining public attention [1,2]. Obesity is the commonest observable indicative of "unhealthiness", leading to cardiovascular problems. Thus, simple objective measurements of obesity like Body Mass Index (BMI), blood lipids and cholesterols are also gaining much public concern [3]. Blood pressure and blood sugar levels naturally fall into the same checklist of safety requirements which are mainly affecting cardiovascular health [4].
As time goes on, the five major influences on cardiovascular health have thus been put together as the "Metabolic Syndrome" (MS): overweight, obesity, hypertension, hyperglycemia and hypercholesterolemia [5]. Another associated pathology, viz. fatty liver, turns up, as many sufferers of MS are found to have different degrees of liver dysfunction and fat deposits gradually leading the way to liver fibrosis [6].

For decades, varieties of interventions are available for the control of the different aspects of MS, particularly in areas 
that require specific effective control like hypertension and hyperglycemia. It is realized now that a better way to deal with the problem is to broaden the area of concern so that MS can be taken together in a multi-facetted preventive endeavor. Although specific single target pharmaceuticals are available for solitary treatment, a more ideal alternative could be some extra-pharmaceutical multiple target, harmonizing supplement to take care of all components of the MS $[7,8]$.

\section{Medicinal Herbs}

A number of Medicinal herbs have been traditionally used as supplement for patients with diabetes mellitus and obesity. They are chosen for our study on metabolic syndrome. Platform studies would include bioactivities related to cardiovascular problems $[9,10]$.

We selected four edible herbals items for the Chinese pharmacopeia to be tested. They are: (Table 1).

Table 1: Four edible herbals items for the Chinese pharmacopeia to be tested.

\begin{tabular}{|c|c|}
\hline Herbal Item & Traditional Use \\
\hline $\begin{array}{c}\text { Crataegus pinnatifida Bge (a tree } \\
\text { berry) }\end{array}$ & Reduce Fat [11] \\
\hline $\begin{array}{c}\text { Schisandra chinensis (Turcz.)(a } \\
\text { scrub berry) }\end{array}$ & Reduce Fat and Protects Liver [12] \\
\hline $\begin{array}{c}\text { Momordica charantia (bitter } \\
\text { melon) }\end{array}$ & Reduce Blood Sugar [13,14] \\
\hline Silymarim Marianum (Milk thistle) & Reduce protects liver [15,16] \\
\hline
\end{tabular}

The combined formula (2MSC) would be tested for the control of body fat and sugar metabolism, while its effects on blood pressure, blood lipids and liver function would carefully be studied [17]. The design of this study on MS has chosen an emphasis on the effects on fatty liver [18].

\section{Methods}

\section{Preclinical Studies}

In vitro experiments included the following:

To investigate specific bioactivities of individual herbs and the combined formula

I. Testing the effects of Crataegus Fructus and the formula on the adipocyte viability and cholesterol uptake using adipocyte and cacocell cultures [19].

II. Testing the effects of Schisandra Fructus and Silymarim Marianum separately and the combined formula on liver cell viability using HepG2 cells [20].

III. Testing the antiglycemic effects of Momordica and the combined formula using Brush Border Membrance Vesicle model via its glucose uptake obstruction [21-23].

\section{In vivo Experiments}

Male C578 1/6 mice were use. Obesity induction was achieved using forced high-fat feeding for 8 weeks. The obese mice were treated with normal diet and continual high fat diet with $2 \%$ and $4 \%$ combined herbal formula [18]. At the end of 8 weeks for the low dose and 12 weeks for the high dose, the animals were sacrificed to have comprehensive checks on Body Weight, Blood examinations for lipid assessments and liver examinations.

\section{Results of Laboratory Studies}

In Vitro Studies

3T3-L1 preadipocytes differentiation cell assay showed the effects of the different concentrations of $125 \mu \mathrm{g} / \mathrm{ml}$, it was a dose that induced significant toxicity to cells, and there was no doseresponse effect observed [19].

Fluorescent tagged cholesterol-treated Caco-2 cell assay

The effect of the different concentrations of Crataegus Fructus aqueous extract and the herbal formula extract on cholesterol uptake in Caco-2 cells. Crataegus Fructus aqueous extract significantly prevented the cholesterol uptake in Caco- 2 cells in a dose dependent manner. Herbal formula extract on the other hand had no significant effect on the cholesterol uptake in Caco- 2 cells [19].

\section{Animal Studies}

The body weight, adipose tissues weight and liver weight were measured. In the 8-week treatment study, the High-fat diet (HF-fed) animals significantly gained more weight than chow-fed animals. Among all HF-fed animals, there was a trend for a reduction in body weight of $4 \%$ among herbal formula fed animals, starting from week 9 onwards. Three types of adipose tissues (epididymal fat, peri-renal fat, and inguinal subcutaneous fat) were isolated and weighed. High-fat diet induced obesity in mice compared to normal chow-fed mice after 16 weeks and 20 weeks, as evidenced by the significant increase in all three types of fat pad mass to body weight ratio: epididymal fat pad $(\mathrm{p}<0.01$ for both 8 -week and 12 -week treatment studies), inguinal fat pad ( $\mathrm{p}<0.01$ for both 8-week and 12 -week treatment studies), and perirenal fat pad ( $p<0.001$ for both 8-week and 12-week treatment studies).

Livers were isolated, weighed and presented as liver to body weight ratio. High-fat diet induced an increase in liver to body weight ratio in both 8-week and 12-week treatment studies [19]. $2 \%$ and $4 \%$ herbal extracts dose-dependently reduced the liver to body weight ratio in both treatment periods.

\section{Liver histopathology and inflammation assessment}

Livers from mice fed on different diets were analyzed histologically. Normal-chow-fed animals demonstrated the 
histological sections of normal livers. In contrast, H\&E sections from $\mathrm{HF}$ animals revealed the presence of a large number of circular lipid droplets within the hepatocytes. These lipid inclusions were clearly reduced in size as well as number in the livers of all herbal formula treated animals.

\section{A Pilot Clinical Trial}

Title: Pilot Clinical Study to evaluate the effects of the innovative herbal formula 2MSC in subjects with Metabolic Syndrome.

Hypothesis: 2 MSC is effective for the Management of MS, with particular emphasis on liver fibrosis (Fatty liver).

\section{Study Design}

An open label pilot study conducted with 30 overweight adult men and women were assigned to take 2 MSC daily for 3 months.

\section{Study Population}

Subjects recruited were 40-66 years of age, with BMI between $\geq 25 \mathrm{~kg} / \mathrm{m}^{2}$ and $\leq 37.7 \mathrm{~kg} / \mathrm{m}^{2}$. They agreed to attend all study visits and to keep their normal dietary habits and usual physical activities. Subjects were excluded if they were diabetic (on diabetic medication for more than four weeks); on cardiac and statin related drugs; on immune-suppressive drugs. Cigarette smokers, pregnant or lactating women were excluded. A total of 30 volunteers with no past history of allergy to herbal medicine were recruited.

\section{Study Procedures}

The study started after signing the proper consent. Duration of treatment lasted 12 weeks. Monthly phone calls were conducted to monitor the progress, compliance and adverse effects. Volunteers were reminded to keep their usual dietary habits and physical activities.

Table 2: Changes of physical measurements of the volunteers.

\begin{tabular}{|c|c|c|c|c|c|c|c|c|c|}
\hline & \multicolumn{3}{|c|}{ Pre-treatment(SD) } & \multicolumn{3}{|c|}{ Post-treatment(SD) } & \multicolumn{3}{|c|}{$P$ value } \\
\hline & Total & Male & Female & Total & Male & Female & Total & Male & Female \\
\hline Age (years) & 53.9 & & & & & & --- & --- & --- \\
\hline $\operatorname{Sex}(M / F)$ & 30 & 11 & 19 & 30 & 11 & 19 & --- & --- & --- \\
\hline BW(kg) & $71.4(11.4)$ & $81.5(10.6)$ & $65.6(6.9)$ & $70.6(11.3)$ & $80.5(10.1)$ & $64.9(7.3)$ & 0.027 & 0.161 & 0.104 \\
\hline BMI & $28.0(2.9)$ & $29.1(3.3)$ & $27.3(2.4)$ & $27.7(3.0)$ & $28.8(3.4)$ & $27.0(2.6)$ & 0.053 & 0.141 & 0.185 \\
\hline Waist $(\mathrm{cm})$ & $97.1(7.2)$ & $102.5(6.1)$ & $93.9(5.9)$ & $94.9(8.1)$ & $101.5(6.0)$ & $91.0(6.6)$ & 0.014 & 0.405 & 0.021 \\
\hline $\operatorname{Neck}(\mathrm{cm})$ & $37.0(3.3)$ & $40.4(2.4)$ & $35.0(1.8)$ & $36.0(3.5)$ & $39.6(2.4)$ & $33.8(1.9)$ & 0.001 & 0.072 & 0.001 \\
\hline $\operatorname{Hip}(\mathrm{cm})$ & $104.5(6.4)$ & $105.8(7.2)$ & $103.7(6.0)$ & $103.3(6.4)$ & $105.1(7.1)$ & $102.2(5.9)$ & 0.011 & 0.407 & 0.01 \\
\hline
\end{tabular}

BW: Body Weight; BMI: body mass index.

The blood checking data indicative of lipid metabolism also showed very positive decline in the triglycerides (Table 3). Fibroscan study showing the "rigidity" of the liver through the anterior abdominal wall, demonstrated softening from 315.3 (49.7) to 291.0 (44.1) p=0.006 (Table 3).

\section{Data Collections}

Demographic and basic measurements related to MS including Body Weight, BMI; Waist Circumference; Hip Circumference and Neck Circumference. Blood testing included fasting blood sugar, Liver function tests, Renal function tests. Other items related to Lipid metabolism included total cholesterol (TC), Triglycerides (TG), Low density (LDL) and high-density lipoprotein cholesterol (HDL), and non-high-density lipoprotein cholesterol. Adiponectin and some immune cytokines were also taken. Fibroscan of the liver was done at baseline and final visit. The Quality of life was checked with the standard SF-36 scoring sheet.

\section{Primary Outcome}

The primary outcome included the decline of Body Weight, Waist Circumference and Lowing of blood triglyceride TG.

\section{Statistical Analysis}

Excel 2016 (Microsoft Corp, Redmond WA) was used for data entry. Statistical analysis was performed using SPSS Base System ver. 25 (IBM SPSS Inc., Chicago IL.). Statistical analysis (descriptive statistics and Student t tests) was performed using SPSS ver. 22 (IBM SPSS Inc., Chicago IL.). Paired t-test was utilized to evaluate the difference between pretreatment mean and post-treatment mean. The percent changes in CAP Reading from baseline to 12 weeks of treatment with 2MSC were analyzed by using Chi-Square test.

\section{Results}

Compliance was excellent. No subject withdrew during study period. Adverse effects reported were all mild, including loose stools and mild abdominal discomfort. Liver and kidney functions remained normal. Bodily measurements all showed clear tendencies of improvement with convincing p values (Table 2). 
Table 3: Changes in the lipid profile and Fibroscan after administration of 2MSC.

\begin{tabular}{|c|c|c|c|c|}
\hline Variable (unit) & Baseline (SD) & 12-week (SD) & Difference* & P value \\
\hline Fasting glucose (mmol/L) & $5.528(1.326)$ & $5.393(1.049)$ & -0.135 & 0.203 \\
\hline TC (mmol/L) & $5.428(0.556)$ & $5.483(0.600)$ & 0.055 & 0.628 \\
\hline LDL (mmol/L) & $3.354(0.438)$ & $3.436(0.482)$ & 0.082 & 0.409 \\
\hline HDL (mmol/L) & $1.314(0.292)$ & $1.376(0.271)$ & 0.062 & 0.034 \\
\hline TG (mmol/L) & $1.941(1.480)$ & $1.645(0.921)$ & -0.297 & 0.044 \\
\hline NonHDL-Cho & $4.145(0.595)$ & $4.079(0.563)$ & -0.066 & 0.526 \\
\hline Fribroscan (CAP) & $313.5(49.1)$ & $291.3(41.9)$ & -22.2 & 0.01 \\
\hline
\end{tabular}

TC, total cholesterol; TG, triglycerides; HDL-C, high-density lipoprotein cholesterol; LDL-C, low-density lipoprotein cholesterol; * 12-week minus Baseline.

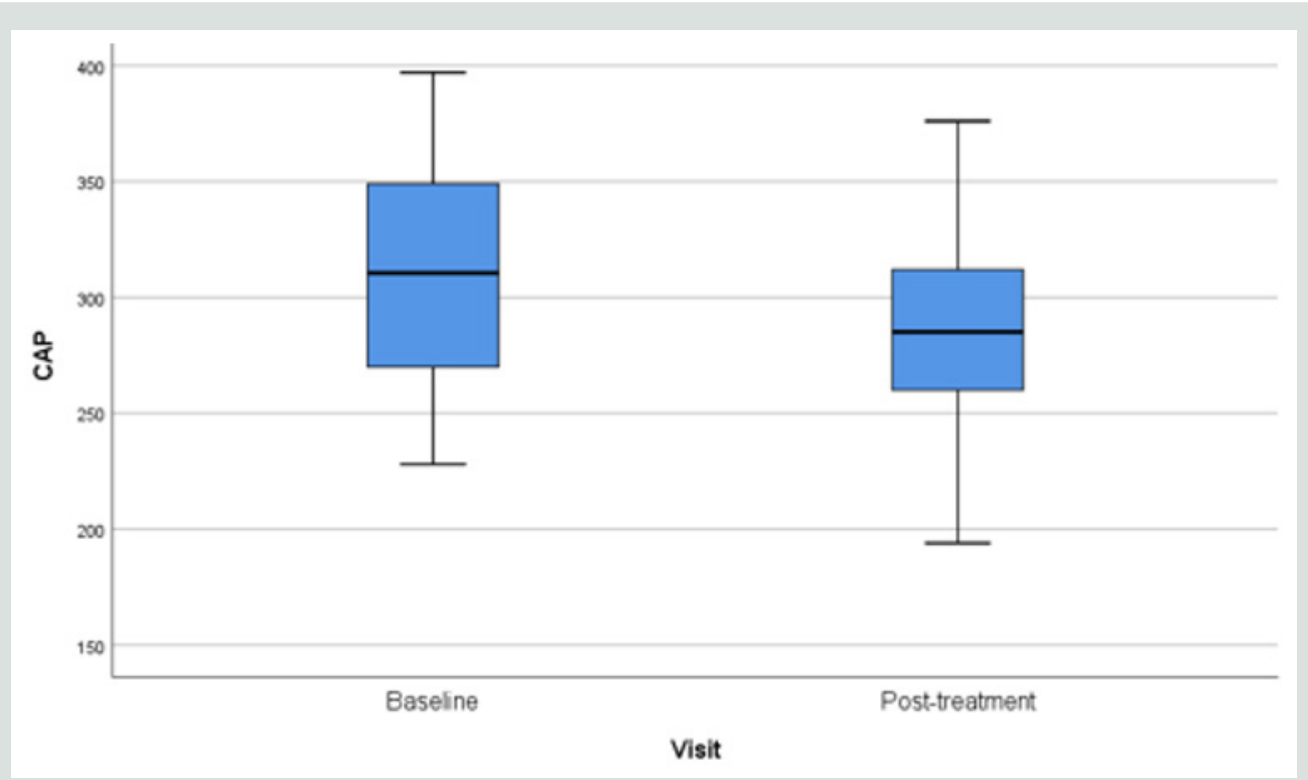

Figure 1: Overall Fibroscan Results: Comparission of controlled attenuation parameter (CAP) values.

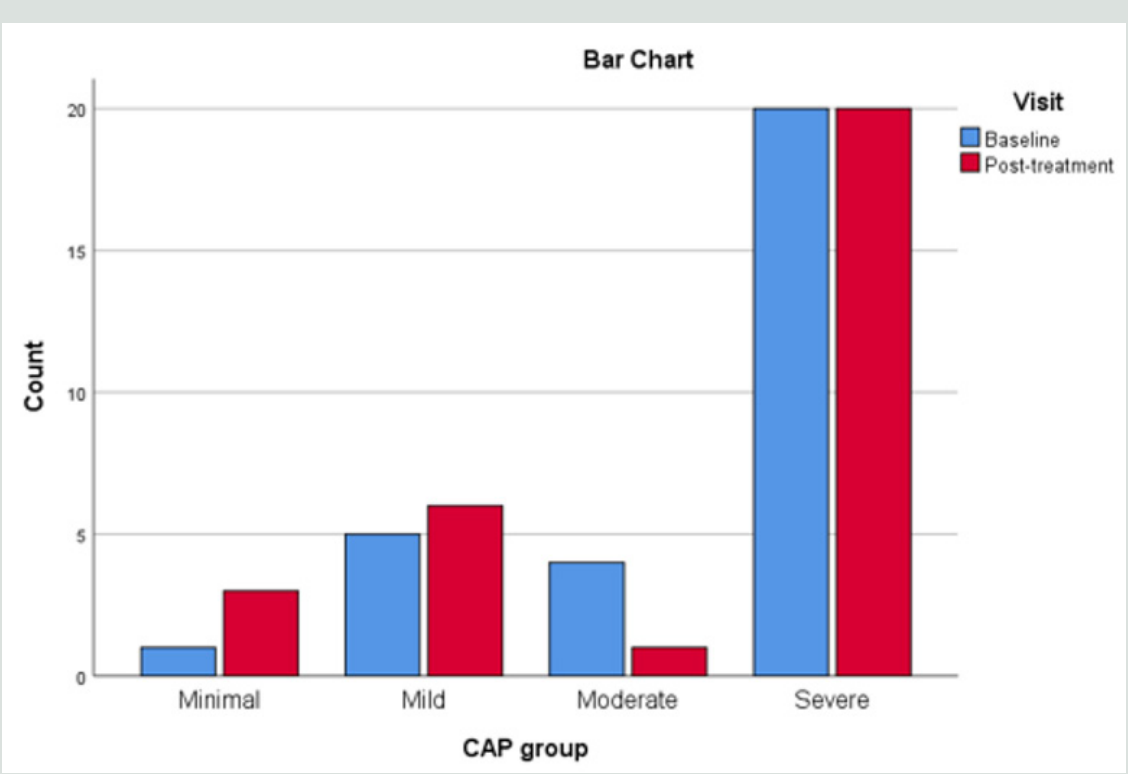

Figure 2: Comparison of CAP Reading. 
When the liver conditions of the volunteers were classified into four different groups of liver steatosis, as: minimal, mild, moderate and severe, it was interesting to find that the herbal formula influenced only the moderate group significantly, while the minimal to mild group and severe group were unaffected. (Figure 2).

\section{Discussion}

Functional foods or nutraceuticals which have potential antiobesity properties have attracted great attention. Schisandrae Fructus is a Chinese herb traditionally used as a liver tonic. Silymarin, an extract of the milk thistle (Silybum marianum), is a dietary supplement that is widely used in Europe for the prevention and treatment of liver problems. Crataegus Fructus (hawthorn) is traditionally used to promote digestion and dissipate food stagnation. Momordica charantia (bitter melon) is traditionally used for the treatment of diabetes in Ayurvedic Medicine. Our in vitro results suggested Crataegus Fructus aqueous extract exerted potent inhibitory effects on 3T3-L1 preadipocytes differentiation and cholesterol uptake into Caco- 2 cells. Schisandrae Fructus aqueous extract and milk thistle exerted inhibitory effects on oleic acid-induced fatty liver in HepG2 cells. Momordica charantia extract on the other hand, exerted significant inhibitory effects on the glucose uptake into BBMV. Our in vivo results showed that our herbal formula exhibited a trend to reduce diet-induced increase in body weight and fat pad mass (epididymal, perirenal and inguinal fat). It also significantly reduced diet-induced increase in liver weight, liver lipid, and plasma lipid dose-dependently. Highfat diet induced a significant reduction in adiponectin level which was significantly improved by herbal formula supplementation at $4 \%$. The herbal formula also significantly reduced diet-induced inflammation in the liver at both doses.

Metabolic Syndrome is currently understood as a combination of commonly co-existing symptoms some of them clearly present uniquely as an outstanding disease, like diabetes mellitus and hypertension, while others present as potentially threatening pathologies. Although adequate treatment for diabetes and hypertension are available, there is a common wish that they could be controlled at an early stage, so that progression could be curbed [23].

Apart from a rigidly followed lifestyle recommended for the cardiovascular system and obesity, which is not easy for the general public, food supplements have been used to help [24,25]. Our pilot clinical study ventured to develop an evidence-based supplement specific for MS, adopting a broad view covering all five problems: obesity, body weight, BMI, blood pressure, and blood lipids. Changes in liver fat and adiponectin were also explored [26,27]. Our pilot clinical study showed an overall body weight loss from 71.4 to $70.6 \mathrm{~kg}(\mathrm{p}=0.027)$ and a BMI 28.0 to 27.7 ( $\mathrm{p}=0.053)$ within a short period of 12 weeks.
The body circumference (external fat collection) shrinkage as detected through waist and hip also showed significant decline ( $\mathrm{p}=0.014$ and 0.011).

Since neck circumference measurement has recently been endorsed as a simple practical assessment for body fat, our neck measurements were supportive to the innovation and showed a 37.0 to $36.0 \mathrm{~cm}$ decline $[28,29]$.

Useful results were provided in the blood tests. Liver function and renal function tests stayed normal, indicating the safety of the formula. With regard to the lipids: there were declines in triglycerages $(p=0.044)$ and an increase in high density lipid $(p=0.034)$ [30]. Fasting blood sugar remained stable.

The overall results were promising but the trial period lasted only 12 weeks and the number of volunteers was small. More than 50 Traditional Chinese Medicine formulae have been used to treat MS, basing on classical theory "Kidney Health", cardiovascular support, and general harmonizing effects [31]. The 4-herbs selected to form the 2MSC formula were taken from classical recommendations subsequently scrutinized on bioactivity platform, to provide pharmacological evidence.

Liver scanning using Ultrasonic Fibroscan device is a simple qualitative and quantitative way to evaluate fat contents in the liver suspected of fatty changes. In our study, liver tissue resistance to Ultrasonic pressure was found to decline from 315.3 to 291.3 $(p=0.010)$ within a period of only 12 weeks (Figure 1) (32-35). There are many reports on the study of non-alcoholic fatty liver in response to supplements [25,36-39] mainly targeting on the liver pathology alone. Our study took a broader view: other factors leading to MS should also be contributing towards fatty changes in the liver.

Since the discovery of Adiponectin as a factorvery much affecting the Metabolism of fatty tissues responsible for the metabolic cycle connecting fat deposit and carbohydrate consumption, the behavior of adiponectin is often included in studies related to MS [40-43]. In our preclinical animal experimental study lasting 12 weeks, we found a high dose of the combined formula gave an increase of adiponectin suggesting that carbohydrates metabolism could have been promoted [19]. In our pilot clinical trial, however adiponectin was found to be either raised, remained stable or decreased. This might be due to the low dose effect or that the study lasting only 12 weeks. Using the SF36 questionnaire for the volunteers' selfevaluation of their physical, mental and social well-being's did not give remarkable results.

\section{Conclusion}

Up to today there is no FDA approved medications for the treatment of non-alcoholic fatty liver disease (NAFLD). The American 
Association for the Study of Liver Diseases (AASLD) suggested the combination use of vitamin E (an antioxidant) and pioglitazone may be helpful but not all patients would benefit from it. For patients diagnosed with NAFLD, the first line of treatment usually involves weight loss through a combination of a healthy diet and exercise. According to the AASLD guidelines, it was recommended that $10 \%$ body weight loss would lead to improvement of the steatosis and inflammation related. Previous studies also found that lifestyle modification could significantly improve the mean fibroscan CAP value $(278.57 \pm 49.13 \mathrm{~dB} / \mathrm{m}$ VS $252.91 \pm 62.02 \mathrm{~dB} / \mathrm{m}, \mathrm{p}=0.03)$. Thus 6 months of lifestyle modification which include moderate intensity physical exercise 3 days per week each for 45 minutes; plus a restricted caloric intake of $25-30 \mathrm{kcals} / \mathrm{kg} /$ day could better protect liver health after tremendous individual efforts and This obviously involves hard work and determination.

Our efforts on the creation of an Evidence-Based Specific Supplement for the control of Metabolic Syndrome have harvested highly positive data in the laboratory and in the subsequent 3 months' pilot clinical trial. Encouraging results were obtained in the control of blood lipids, general body measurements and liver fibrosis. It is envisaged that coupled with more exercises and dietary control, better results could be expected. Further clinical studies would be very much warranted.

\section{Acknowledgement}

This project was financially supported by The Innovation \& Technology Fund (Ref. no ITS/036/15FX) of the Innovation \& Technology Commission of Hong Kong SAR, Venture Tycoon Limited, The State Key Laboratory of Research on Bioactivities and Clinical Applications of Medicinal Plants (The Chinese University of Hong Kong) and The Royal Medic Research and Development Center, Hong Kong Science Park, Hong Kong.

\section{References}

1. Huang PL (2009) A comprehensive definition for metabolic syndrome. Dis Model Mech 2(5-6):231-237.

2. Kaur J (2014) A comprehensive review on metabolic syndrome. Cardiol Res Pract 2014: 943162.

3. Ford ES (2005) Prevalence of the metabolic syndrome defined by the International Diabetes Federation among adults in the US. Diabetes Care 28(11): 2745-2749.

4. Binesh Marvasti T, Adeli K (2010) Pharmacological management of metabolic syndrome and its lipid complications. Daru 18(3): 146-154.

5. Deedwania PC, Gupta R (2006) Management issues in the metabolic syndrome. J Assoc Phys India 54: 797-810.

6. Farrell GC, Larter CZ (2006) Nonalcoholic fatty liver disease: from steatosis to cirrhosis. Hepatology 43 (2 Suppl 1): S99-S112.

7. Grundy SM (2007) Metabolic syndrome: a multiplex cardiovascular risk factor. J Clin Endocrinol Metab 92(2): 399-404.

8. Monsour HP, Frenette CT, Wyne K (2012) Fatty Liver: A Link to Cardiovascular Disease - Its Natural History, Pathogenesis, and Treatment. Methodist Debakey Cardiovasc J 8(3): 21-25.
9. Yang J, Zhang HB (2011) Profile and primary investigation of Traditional Chinese Medicine in treatment of hyperlipidemia. Shan Xi Zhong Yi 32: 260-261.

10. Matfin G (2010) Developing therapies for the metabolic syndrome: challenges, opportunities, and... the unknown. Ther Adv Endocrinol Metab 1(2): 89-94.

11. Hu M, Zeng W, Tomlinson B (2014) Evaluation of a crataegus-based multiherb formula for dyslipidemia: a randomized, double-blind, placebo-controlled clinical trial. Evid Based Complement Alternat Med 365742 .

12. O'Brien KA (2010) Alternative perspectives: how Chinese Medicine understands hypercholesterolemia. Cholesterol 723289 Pp: 1-9.

13. Fuangchan A, Sonthisombat P, Seubnukarn T, Chanouan $R$, Chotchaisuwat P, et al. (2011) Hypoglycemic effect of bitter melon compared with metformin in newly diagnosed type 2 diabetes patients. J Ethnopharmacol 134(2): 422-428.

14. Jayasooriya AP, Sakono M, Yukizaki C, Kawano M, Yamamoto K, et al. (2000) Effects of Momordica charantia powder on serum glucose levels and various lipid parameters in rats fed with cholesterol-free and cholesterol-enriched diets. J Ethnopharmacol 72(1-2): 331-336.

15. Pais P, D'Amato M (2014) In vivo efficacy study of milk thistle extract (ETHIS-094) in STAM model of nonalcoholic steatohepatitis. Drugs R D 14(4): 291-299.

16. Lee JI, Hsu BH, Wu D, Barrett JS (2006) Separation and characterization of silybin, isosilybin, silydianin and silychristin in milk thistle extract by liquid chromatography-electrospray tandem mass spectrometry. J Chromatogr A 1116(1-2): 57-68.

17. Kuo DH, Yeh CH, Shieh PC, Cheng KC, Chen FA, et al. (2009) Effect of Shanzha, a Chinese herbal product, on obesity and dyslipidemia in hamsters receiving high-fat diet. J Ethnopharmacol 124(3): 544-550.

18. Wat E, Tandy S, Kapera E, Kamili A, Chung RW, et al. (2009) Dietary phospholipid-rich dairy milk extract reduces hepatomegaly, hepatic steatosis and hyperlipidemia in mice fed a high-fat diet. Atherosclerosis 205(1): 144-150.

19. Wat E, Wang YP, Ken Chan, Hon Wai Law, Chi Man Koon, et al. (2018) An in vitro and in vivo study of a 4-herb formula on the management of dietinduced metabolic syndrome. Phytomedicine 42: 112-125.

20. Ge ZZ, Nie R, Maimaiti T, Yao F, Li C (2016) Comparison of the inhibition on cellular 22-NBD-cholesterol accumulation and transportation of monomeric catechins and their corresponding A-type dimers in Caco-2 cell monolayers. J Funct Foods 27: 343-351.

21. Kessler M, Acuto O, Storelli C, Murer H, Müller M, et al. (1978) A modified procedure for the rapid preparation of efficiently transporting vesicles from small intestinal brush border membranes. Their use in investigating some properties of Dglucose and choline transport systems. Biochim Biophys Acta 506(1): 136-154.

22. Hopfer U, Nelson K, Perrotto J, Isselbacher KJ (1973) Glucose transport in isolated brush border membrane from rat small intestine. J Biol Chem 248(1): 25-32.

23. Polyzor SA, Mantzoros CN (2016) Nonalcholic fatty future disease. Metabolism 65(8): 1007-1016.

24. Zhang HJ, Pan LL, Ma ZM, Chau Z (2017) Longterm effect of exercise on improving fatty liver and cardiovascular risk factors in obese adults: A 1-year follow-up study. Diabetes Obs Metab 19(2): 284-289.

25. Moon HS, Chung CS, Lee HG, Kim TG, Choi YJ, et al. (2007) Inhibitory effect of (-)-epigallocatechin-3-gallate on lipid accumulation of 3T3-L1 cells. Obesity (Silver Spring) 15(11): 2571-2582.

26. Qihe Xu, Yuen Fei Wong, Shanshan Qu, Qingyang Kong, Xiu-Li Zhang, et al. (2010) Knowledge-based discovery of antifibrotic and pro-fibrotic 
activities from Chinese Materia Medica. Recent advances in Theories and Practice of Chinese Medicine 337-351.

27. Polyzos SA, Kountouras J, Mantzoross (2016) Adipokines in nonalcoholic fatty liver disease. Metabolism 65(8): 1062-1079.

28. Hassan Salmanroghani, Roham Salmanroghani, Mahyar Nourian, Karim Khayarn, Farhad Lahmi, et al. (2019) Evaluation of Neck Circumference as an easy and reliable predictor of NAFLD. Turk J Gastroenterol 30(2):163-170

29. Huang BX, Zhu MF, Wu Ting, Jing-ya Zhou, Yan Liu, et al. (2015) Neck Circumference, along with Other Anthropometric Indices, Has an Independent and Additional Contribution in Predicting Fatty Liver Disease. PLoS One 10(2): e0118071.

30. Farrell G, Larter CZ (2006) Nonalcoholic fatty liver disease: From steatosis to cirrhosis. Liver Failure and Liver Disease. Hepatology vol 43(2): S99-S111.

31. Lau CH, Chan CM, Chan YW, Lau KM, Lau TW, et al. (2007) Pharmacological investigations of the anti-diabetic effect of Cortex Moutan and its active component paeonol. Phytomedicine 14(11): 778-784.

32. Ratziu V, Giral P, Charlotte F, E Bruckert, V Thibault, et al. (2000) Liver fibrosis in overweight patients. Gastroenterology 118(6): 1117-1123.

33. Angulo P, Hui JM, Marchesini G, et al. (2007) The NAFLD fibrosis score: a noninvasive system that identifies liver fibrosis in patients. Hepatology 45(4): 846-854.

34. Jeong-Hoon Lee, Donghee Kim, Hwa Jung Kim, Chang-Hoon Lee, Jong In Yang, et al. (2010) Hepatic steatosis index: a simple screening tool reflecting non-alcoholic fatty liver disease. Dig Liver Dis 42(7): 503-508.

35. Frederik Cornelis Kruger, Caroline Rachel Daniels, Martin Kidd, Gillaum Swart, Karen Brundyn, et al. (2011) A simple bedside marker for advanced fibrosis that can avoid liver biopsy in patients with NAFLD. S Afr Med J 101(7): 477-480.

36. Wagh A, Stone NJ (2004) Treatment of metabolic syndrome. Expert Rev Cardiovasc Ther 2(2): 213-228.

37. De Ligt M, Timmers S, Schrauwen P (2015) Resveratrol and obesity: Can resveratrol relieve metabolic disturbances? Biochim Biophys Acta 1852(6):1137-1144.

38. Ahmed Elgebaly, Ibrahim A I Radwan, Mohamed M AboElnas, Hamza H Ibrahim, Moutaz F M Eltoomy, et al. (2017) Resveratrol Supplementation in Patients with Non-Alcoholic Fatty Liver Disease: Systematic Review and Meta-analysis. Journal of gastrointestinal and liver diseases 26(1):59-67.

39. Silvie Timmers, Marlies de Ligt, Esther Phielix, Tineke van de Weijer, Jan Hansen, et al. (2016) Resveratrol as Add-on Therapy in Subjects with Well-Controlled Type 2 Diabetes: A Randomized Controlled Trial. Diabetes Care 39(12): 2211-2217.

40. Frankenberg ADV, Reis AF, Gerchman F (2017) Relationships between adiponectin levels, the metabolic syndrome, and type 2 diabetes: a literature review. Arch Endocrinol Metab 61(6): 614-622.

41. Han Fang, Judd RL (2018) Adiponectin Regulation and Function. Compr Physiol 8(3): 1031-1063.

42. Miwa Ryo, Tadashi Nakamura, Shinji Kihara, Masahiro Kumada, Satomi Shibazaki, et al. (2004) Adiponectin as a Biomarker of the Metabolic Syndrome. Circulation Journal 68(11): 975-981.

43. Ghadge AA, Khaire AA, Kuvalekar AA (2018) Adiponectin: A potential therapeutic target for Metabolic Syndrome. Cytokine Growth Factor Rev 39: 151-158.
This work is licensed under Creative Commons Attribution 4.0 License

\section{To Submit Your Article Click Here: $\quad$ Submit Article}

DOI: $10.32474 / A D 0.2020 .03 .000158$

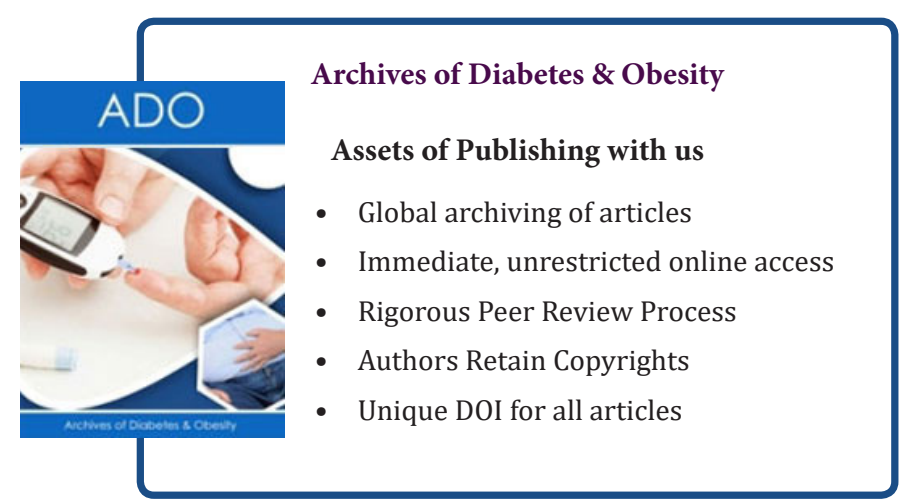

